\title{
Erprobung und Akzeptanz eines psychosozialen Screenings in der primärärztlichen und orthopädischen Versorgung zur Steuerung des Rehabilitationszugangs bei muskuloskelettalen Erkrankungen
}

\section{Implementation and Acceptance of a Psychosocial Screening in Primary und Orthopedic Care to Manage Rehabilitation Access in Musculoskeletal Diseases}

Autoren

Stefanie Schmidt ${ }^{1}$, Franziska-Antonia Zora Samos ${ }^{2}$, Andreas Klement ${ }^{2}$, Julia-Marie Krüger ${ }^{1}$, Wilfried Mau ${ }^{1}$

Institute

1 Institut für Rehabilitationsmedizin, Medizinische Fakultät der Martin-Luther-Universität Halle-Wittenberg, Halle (Saale)

2 Institut für Allgemeinmedizin, Medizinische Fakultät der Martin-Luther-Universität Halle-Wittenberg, Halle (Saale)

\section{Schlüsselwörter}

Muskuloskelettale Erkrankungen, psychische Komorbidität, Ultra-Kurz-Screening, psychometrische Eigenschaften, Akzeptanzanalyse

\section{Key words}

musculoskeletal diseases, mental comorbidity, ultra-shortscreening, psychometric properties, acceptance analysis

eingereicht 10.10 .2018

akzeptiert 28.01.2019

Bibliografie

DOI https://doi.org/10.1055/a-0842-6901

Online-Publikation: 2.5.2019

Phys Med Rehab Kuror 2019; 29: 206-214

(c) Georg Thieme Verlag KG Stuttgart · New York ISSN 0940-6689

Korrespondenzadresse

Dr. Stefanie Schmidt

Institut für Rehabilitationsmedizin, Medizinische Fakultät der Martin-Luther-Universität Halle-Wittenberg

Magdeburger Straße 8

06097 Halle (Saale)

stefanie.schmidt@medizin.uni-halle.de

\section{ZUSAMMENFASSUNC}

Hintergrund Für muskuloskelettale Erkrankungen konnte in zahlreichen Studien eine hohe psychische Komorbidität festgestellt werden. Aufgrund der für die PatientInnen oftmals im Vordergrund stehenden somatischen Beschwerden werden psychische Belastungen im Behandlungsprozess und bei der
Reha-Antragstellung vielfach nicht erkannt bzw. in Befundberichten nicht benannt. Dementsprechend fehlen behandelnden ÄrztInnen häufig Informationen und dem Leistungsträger bei der Reha-Antragsbewertung und nachfolgenden Klinikauswahl angemessene Entscheidungsgrundlagen. Daraus ergibt sich die Notwendigkeit der Erprobung eines differenzierten Screenings für psychische Problemlagen im Vorfeld der Rehabilitation.

Material und Methoden In der vorliegenden clusterrandomisierten Studie sollten niedergelassene ÄrztInnen aus hausärztlichen und orthopädischen Praxen aus Mitteldeutschland (Sachsen, Sachsen-Anhalt und Thüringen) ihre PatientInnen bei Antrag auf Rehabilitation bitten, den üblichen Antragsunterlagen zusätzlich einen ausgefüllten Ultra-Kurz-Screening-Fragebogen (UKS) für die PrüfärztInnen der Rentenversicherung beizulegen. Neben soziodemografischen und rehabilitationsbezogenen Parametern wurden gesundheitsbezogene Merkmale von insgesamt 119 PatientInnen erhoben. Zusätzlich wurden Analysen zur Reliabilität und Validität des UKS durchgeführt. Des Weiteren wurden die Zuweisungsentscheidungen der PrüfärztInnen dokumentiert. Außerdem wurde den teilnehmenden PraxisärztInnen $(n=106)$ als auch allen PrüfärztInnen der Rentenversicherung ( $n=42)$ ein Fragebogen vorgelegt, um ihre Einschätzung zu psychosozialem Screening im Rahmen der Reha-Antragstellung und -entscheidung zu erfahren.

Ergebnisse Im UKS zeigten sich bei der Mehrheit der StudienteilnehmerInnen zum Zeitpunkt der Reha-Antragstellung Hinweise auf moderate psychosoziale Problemlagen (69\%), bei guten psychometrischen Werten für Reliabilität und Validität im Vorfeld der Rehabilitation. Von der Mehrheit (78\%) der Vertrags- und PrüfärztInnen wurde die routinemäßige Implementierung des UKS in die Reha-Antragstellung bei MSK befürwortet.

Diskussion Zusammenfassend erscheint es sinnvoll, psychosoziale Belastungen standardisiert im Rahmen der Reha-Antragstellung zu erfassen, damit behandelnden und antragsbearbeitenden ÄrztInnen diese zusätzliche Information zur bedarfsgerechten Auswahl und Zuweisung geeigneter Interventionen und Rehabilitationskonzepte vorliegt. Eine Integration des UKS in die Routineanwendung sollte unter Beachtung 
der in dieser Studie gewonnenen Erkenntnisse weiter evaluiert werden.

\section{ABSTRACT}

Background In numerous studies, increased mental co-morbidity has been observed for musculoskeletal diseases. Due to the somatic conditions often predominating for the patients, psychological strain in the treatment and application process for medical rehabilitation may not be recognized and documented in diagnostic reports. Therefore, physicians have a lack of information and service providers cannot consider suitable rehabilitation concepts for the selection of rehabilitation clinics. As a result, it is necessary to conduct a screening for psychological problems before rehabilitation.

Materials and Methods In this cluster-randomized study, doctors from general and orthopedic practices from Central Germany (Saxony, Saxony-Anhalt and Thuringia) should instruct their patients to additionally submit the Ultra-Short Screening Questionnaire (UKS) for the medical assessors of the German pension insurance when applying for rehabilitation. In addition to sociodemographic and rehabilitation-related parameters, health-related characteristics were collected from 119 pa- tients. Additionally, analyses of the reliability and validity of the UKS were also carried out. Furthermore, the assignment decisions of the medical assessors were documented and a questionnaire was submitted to the participating practitioners $(n=106)$ as well as to all medical assessors of the German pension insurance $(n=42)$ regarding their evaluation of the psychosocial screening in the process of the rehabilitation application and decision.

Results As a result of the screening, the majority of the study participants showed indications of moderate psychosocial stress at the time of application for rehabilitation (69\%). In addition, the UKS had good psychometric values for reliability and validity before rehabilitation. The majority (78\%) of general and orthopedic practitioners as well as medical assessors support the routine implementation of the UKS when applying for rehabilitation.

Disussion It is sensible to standardize the recording of psychosocial stress when applying for rehabilitation in order to provide all involved physicians with this additional information for the demand-oriented allocation of suitable interventions and rehabilitation concepts. The integration of UKS into routine care should be further evaluated taking into account the findings of this study.

\section{Einleitung}

Zu den häufigsten chronischen Erkrankungen gehören muskuloskelettale Erkrankungen (MSK), deren Versorgung eine zunehmende Herausforderung für die Gesundheitssysteme der westlichen Industrieländer darstellt, v. a. Krankheiten der Wirbelsäule und des Rückens (Dorsopathien) sowie entzündliche und degenerative Gelenkerkrankungen (Arthropathien) [1]. Die Punktprävalenz von Rückenschmerzen ohne detaillierte Angaben zum Schweregrad der Beeinträchtigungen liegt bundesweit zwischen 32 und 49\% (Altersgruppe 18-75) [1, 2]. Bei einer Befragung von ausschließlich erwerbstätigen Personen in der Altersgruppe 40-54 zeigte sich sogar eine Prävalenzrate von 68\% [3]. Mit mehr als einem Drittel aller Rehabilitationsleistungen der Deutschen Rentenversicherung sind MSK weiterhin die häufigste Indikation [4].

Neben einem breiten Spektrum an funktionalen Einschränkungen erleben Betroffene mit einer MSK häufig psychosoziale Belastungen [5]. Mit einer Punktprävalenz von 31 \% weisen RehabilitandInnen wegen einer MSK eine hohe Prävalenz behandlungsbedürftiger psychischer Belastungen auf [6]. Sie führen zu erheblichen sozioökonomischen Konsequenzen [7] mit ungünstigen sozialmedizinischen Verläufen, wie langen Arbeitsunfähigkeitszeiten und hohen Anteilen an Erwerbsminderungsrentenantragstellenden [810].

Im Rahmen einer medizinischen Rehabilitation der Deutschen Rentenversicherung als häufigsten Leistungsträger können für die PatientInnen mit MSK je nach Ausprägung der psychischen Problemlagen verschiedene Rehabilitationskonzepte realisiert werden: die orthopädische Rehabilitation (OR), die verhaltensmedizinischorthopädische (VMO) oder die psychosomatische Rehabilitation
(PR). Die VMO gilt als multiprofessioneller Behandlungsansatz mit psychosozialer Schwerpunktsetzung [11] und fokussiert neben schmerzbezogenen Verhaltensweisen und Kognitionen auch vorliegende psychische Komorbidität [12]. Zwar führen einige Rehabilitationskliniken eine ausführliche psychologische Diagnostik zu Beginn der Rehabilitation durch, jedoch ist der Rehabilitationsbeginn dieser Leistungen ein zu später Zeitpunkt zur Erfassung gesundheitsrelevanter Beeinträchtigungen, da die Entscheidung zur Konzeptauswahl der Rehabilitation in der Regel vom Leistungsträger bzw. den sozialmedizinischen Prüf- und GutachterärztInnen (nachfolgend PrüfärztInnen genannt) bereits getroffen wurde. Die Konzeptauswahl kann zu Rehabilitationsbeginn kaum revidiert werden, erst recht nicht, wenn die ausgewählte Klinik keine geeigneten Konzepte, wie z. B. die VMO, für die PatientInnen anbieten kann. In erster Linie richtet sich die Konzeptauswahl nach der aus den Antragsunterlagen hervorgehenden führenden Rehabilitationsindikation (ICD-Diagnose). Da für Betroffene meist die somatischen Beschwerden im Vordergrund stehen [13], kommt es oftmals vor, dass psychische Störungen nicht wahrgenommen und daher spät oder gar nicht im Behandlungsprozess erkannt werden [14]. Verschiedene Studien aus der Rehabilitationsforschung belegen, dass hier die Erkennungsrate für psychische Störungen unter $50 \%$ liegt $[15,16]$. Die Identifikation von komorbiden psychischen Störungen findet oftmals spät, erst während der Rehabilitation, statt und wird unzureichend dokumentiert $[17,18]$.

Zur Identifikation psychischer Belastungen im Vorfeld einer medizinischen Rehabilitation gibt es derzeit keine zufriedenstellenden, einheitlichen assessmentbasierten Zuweisungsmechanismen durch die Leistungsträger. Die Studie von Schwarz et al. [19] überprüfte die Eignung der Indikationskriterien bei der Zuweisung zur 
orthopädischen bzw. verhaltensmedizinisch-orthopädischen Rehabilitation und konnte zeigen, dass es eine geringe Übereinstimmung zwischen den Urteilen der sozialmedizinischen PrüfärztInnen und den Rehabilitationskliniken hinsichtlich der Zuweisungsentscheidung auf Grundlage dieser Kriterien gibt.

In der vorliegenden Studie wurde mit Förderung der Deutschen Rentenversicherung Mitteldeutschland (DRV MD) der Einsatz eines psychosozialen Ultra-Kurz-Screening (UKS) [20] im Rahmen der Zuweisungssteuerung zur medizinischen Rehabilitation im Zuge der Reha-Antragstellung aus Haus- und Facharztpraxen erprobt. Das UKS wurde ursprünglich zur Identifizierung psychosozialer Problemlagen bei orthopädischer Rehabilitationsindikation entwickelt und hatte sich bereits als Instrument der Zuweisungssteuerung von Interventionen innerhalb somatisch ausgerichteter Rehabilitationskliniken bewährt [21, 22]. Daher ist ein zentrales Ziel der Studie die Überprüfung der Eignung des UKS als Element der Zuweisungssteuerung im Zuge der Reha-Antragstellung und -Bearbeitung bei MSK. Weiteres Ziel ist die Analyse der Akzeptanz des UKS von ÄrztInnen der hausärztlichen oder ambulanten orthopädischen Versorgung, da es diesbezüglich dieser Aspekte bisher nur wenige, bisher positive Untersuchungen gibt [23]. Dazu sollen folgende Fragestellungen beantwortet werden:

1. Wie häufig sind psychosoziale Problemlagen bei Patientlnnen mit MSK, die in der hausärztlichen oder ambulanten orthopädischen Versorgung in der Region Mitteldeutschland einen Reha-Antrag stellen?

2. Welche psychometrischen Eigenschaften weist das UKS bei der Erfassung psychosozialer Belastungen im Vorfeld der Rehabilitation auf?

3. Wie oft entscheiden sich antragsbearbeitende Prüfärztlnnen der DRV MD für eines der 3 Rehabilitationskonzepte (OR, VMO und PR), wenn das UKS für psychosoziale Problemlagen den üblichen Reha-Antragsunterlagen beiliegt und wenn es diesen Unterlagen nicht beiliegt?

4. Wie wird der Einsatz des UKS im Zuge der Reha-Antragstellung zur medizinischen Rehabilitation von ÄrztInnen der primärärztlichen und orthopädischen Versorgung sowie antragsbearbeitenden PrüfärztInnen der DRV MD eingeschätzt?

\section{Material und Methodik}

\section{Studiendesign}

Im Rahmen dieser clusterrandomisierten, kontrollierten Studie wurde in Haus- und Facharztpraxen für Orthopädie (Studienpraxen) in der Region Mitteldeutschland (Sachsen, Sachsen-Anhalt, Thüringen) der Einsatz des UKS bei Reha-AntragsstellerInnen mit MSK erprobt.

\section{Praxis- und Patientenrekrutierung}

Aufgrund vorangegangener telefonischer Fallhäufigkeitsangaben bei sechs verschiedenen Haus- und Facharztpraxen in der Region sollten insgesamt 90 Ärztlnnen (ein Drittel Hausärztlnnen, zwei Drittel OrthopädInnen) 540 PatientInnen (6 PatientInnen pro Praxis) innerhalb von 6 Monaten in die Studie einschließen. Per Zufallsziehung der Praxisadressen aus öffentlich zugänglichen Datenbanken der Kassenärztlichen Vereinigungen sowie diverser Studien- aufrufe von Stakeholdern der Allgemeinmedizin und Orthopädie in den 3 Bundesländer wurden die Arztpraxen schriftlich über die Studie informiert und um ihre Teilnahme gebeten. Nach dem ersten Studienaufruf erfolgte nur ein geringer Rücklauf (5\%), daher wurde die Anzahl der angeschriebenen Praxen in einer zweiten Rekrutierungswelle deutlich erhöht und mit zahlreichen telefonischen Nachfragen unterstützt. Insgesamt wurden dabei 1346 Arztpraxen kontaktiert. Nach Studieneinschluss von letztendlich 106 teilnahmebereiten Praxen (Rücklaufquote von $8 \%$ ) wurden diese zunächst in Kontroll- bzw. Interventionsgruppe (KG/IG) clusterrandomisiert und erhielten anschließend einen strukturierten Studienordner mit allen Informationen und Materialien. Auf Wunsch wurde eine zusätzliche telefonische Schulung der Studienpraxen durchgeführt. Für vollständige Patientendaten erhielten die Studienkoordinatorlnnen der Praxen Incentives in Form von selbst gewählten Markengutscheinen im Wert von 20 EUR pro vollständig dokumentiertem/r Patientln.

\section{Datenerhebung}

Im Zeitraum 06/2016 bis 06/2017 wurden 119 PatientInnen im Alter zwischen 18 und 65 Jahren mit einer ICD-10-Diagnose aus dem Bereich „Krankheiten des Muskel-Skelett-Systems und des Bindegewebes“ (M00-99), die bei der DRV MD versichert waren, als TeilnehmerInnen in die Studie eingeschlossen.

Zum ersten Messzeitpunkt (T0) füllten alle Studienteilnehmerlnnen ergänzend zum Reha-Antrag das UKS in der Studienpraxis aus. In der Kontrollgruppe (KG) gingen beim Leistungsträger die üblichen Reha-Antragsunterlagen ein. TeilnehmerInnen aus der Interventionsgruppe (IG) wurden gebeten, eine Version des ausgefüllten UKS den Reha-Antragsunterlagen für die Prüfärztlnnen der DRV MD beizulegen. Das UKS wurde von den PrüfärztInnen mittels Auswertungsschablone im Rahmen der Reha-Antragsbearbeitung ausgewertet. Eine Kopie des UKS ging in beiden Studiengruppen pseudonymisiert an das Studienzentrum. In beiden Studiengruppen dokumentierten die PrüfärztInnen außerdem, ob der Reha-Antrag bewilligt oder abgelehnt wurde. Außerdem sollte evaluiert werden, ob die vorliegenden Angaben des UKS für eine Entscheidungsfindung bei der Konzeptauswahl von Bedeutung sind. Im Falle der Bewilligung wurde auch das zugewiesene Rehabilitationskonzept (OR, VMO, PR) notiert und ebenfalls an das Studienzentrum übermittelt. Alle StudienteilnehmerInnen wurden zum Zeitpunkt des Leistungsbescheides als zweiten Messzeitpunkt (T1) gebeten, das UKS wiederholend in einem ausführlichen standardisierten Fragebogen zusammen mit soziodemografischen, gesundheits- sowie rehabilitationsbezogenen und sozialmedizinischen Aspekten auszufüllen.

Das UKS besteht aus insgesamt neun Items, von denen 4 Items aus dem PHQ-4-Screeninginstrument Hinweise auf Depressivität und Ängstlichkeit erfassen [24, 25]. Zwei weitere Items erfragen die subjektive Belastung durch körperliche Schmerzen. Zusätzlich erfassen 2 Items die gegenwärtigen besonderen Belastungen in Familie und Beruf. Ein neuntes Item, welches nicht in die Testauswertung eingeht, erfragt die Wichtigkeit psychologischer Therapieinhalte während der eigenen Rehabilitation. Für die Auswertung wurden für die Konstrukte „Depressivität“, „Ängstlichkeit“ sowie die „Schmerzbelastung“ Subskalen aus den Mittelwerten berechnet, die mit den Werten der Items zur „beruflichen“ und „familiären Belastung“ zu einem Gesamtwert aufsummiert werden (UKS-Ge- 
- Tab. 1 Beschreibung der Stichprobe.

\begin{tabular}{|c|c|c|c|c|c|}
\hline & & & \multicolumn{3}{|c|}{$M( \pm S D) /(\%)$} \\
\hline & & $\mathbf{n}$ & $\begin{array}{l}\text { Gesamt } \\
(n=83)\end{array}$ & KG $(n=36)$ & IG $(n=47)$ \\
\hline \multicolumn{2}{|l|}{ Alter (in Jahren) } & 83 & $53,4( \pm 7,5)$ & $52,5( \pm 8,7)$ & $54,1( \pm 6,4)$ \\
\hline \multicolumn{2}{|l|}{ Frauen } & 44 & $53 \%$ & $58 \%$ & $49 \%$ \\
\hline \multirow[t]{4}{*}{ Familienstand } & ledig & 10 & $11 \%$ & $11 \%$ & $13 \%$ \\
\hline & verheiratet & 52 & $67 \%$ & $67 \%$ & $61 \%$ \\
\hline & getrennt/geschieden & 15 & $17 \%$ & $17 \%$ & $20 \%$ \\
\hline & verwitwet & 5 & $6 \%$ & $6 \%$ & $6 \%$ \\
\hline \multirow[t]{3}{*}{ höchster Schulabschluss } & Volksschul-/Hauptschulabschluss & 6 & $9 \%$ & $9 \%$ & $6 \%$ \\
\hline & $\begin{array}{l}\text { Realschulabschluss/Mittlere Reife/Polytechnische } \\
\text { Oberschule }\end{array}$ & 61 & $63 \%$ & $63 \%$ & $83 \%$ \\
\hline & Fachhochschulreife/Abitur & 14 & $29 \%$ & $29 \%$ & $9 \%$ \\
\hline \multirow[t]{3}{*}{ Berufliche Stellung } & Arbeiter(in) & 35 & $25 \%$ & $25 \%$ & $59 \%$ \\
\hline & Angestellte(r) & 41 & $75 \%$ & $75 \%$ & $37 \%$ \\
\hline & Sonstige & 2 & - & - & $2 \%$ \\
\hline \multicolumn{2}{|l|}{ erwerbstätig } & 66 & $80 \%$ & $80 \%$ & $80 \%$ \\
\hline \multicolumn{2}{|l|}{ arbeitsunfähig (AU) } & 34 & $41 \%$ & $39 \%$ & $43 \%$ \\
\hline \multicolumn{2}{|c|}{ AU-Tage/Monat bezogen auf die letzten 12 Monate } & 60 & $8,1( \pm 8,4)$ & $7,4( \pm 8,3)$ & $8,6( \pm 8,5)$ \\
\hline \multicolumn{2}{|c|}{ Dauer der Erkrankung (in Jahren) } & 78 & $7,4( \pm 8,5)$ & $7,5( \pm 9,3)$ & $7,2( \pm 7,9)$ \\
\hline
\end{tabular}

samtscore: 0-12). Folgende Cut-Off-Werte wurden für die Interpretation des Gesamtscores gemäß der Autorenangabe herangezogen [21]: $\leq 4$ Punkte „ganz oder eher unauffällig“, 5-8 Punkte „moderat ausgeprägte psychosoziale Belastung“, 9-12 „stark oder sehr stark ausgeprägte psychosoziale Belastung“. Zur Erfassung der psychischen Komorbidität wurde neben dem UKS [20] außerdem das Brief Symptom Inventory (BSI-18) [26] eingesetzt. Das BSI18 ist ein 18 Items umfassendes Instrument und erfasst mit jeweils sechs Items das Ausmaß an „Ängstlichkeit“, „Depressivität“ und „Somatisierung“. Zudem kann über alle Subskalen hinweg ein globaler Kennwert der psychischen Belastung (Global Severity Index) bestimmt werden.

Nach Abschluss der Patientenbefragung (T2) wurden sowohl die StudienärztInnen als auch die PrüfärztInnen der DRV MD gebeten, einen standardisierten Fragebogen zur Akzeptanz und Einschätzung der zukünftigen Umsetzung des UKS, der dem Fragebogen als Anlage beilag, im Rahmen der Reha-Antragstellung auszufüllen.

\section{Datenauswertung}

Zur Beschreibung kontinuierlicher Merkmale wurden Mittelwerte (M) und Standardabweichungen (SD), für kategoriale Merkmale Prozentwerte (\%) berechnet. Gruppenunterschiede in kontinuierlichen Merkmalen zwischen 2 Gruppen wurden anhand von t-Tests für unabhängige Stichproben geprüft, Gruppenunterschiede kategorialer Merkmalen wurden mittels Chi-Quadrat-Tests (McNemar), auch zwischen 2 Zeitpunkten, überprüft. Zur Analyse der psychometrischen Eigenschaften des UKS zu T0 bzw. T1 wurden Reliabilitäts- und Validitätsanalysen (interne Konsistenz, Mittelwertdifferenz, bivariate Korrelation) mit Teilnehmerdaten durchgeführt, deren Reha-Antrag positiv beschieden wurde. Die Auswertung der Zuweisungsdokumentation innerhalb der DRV erfolgte mittels deskriptiver Analysen. Das Signifikanzniveau wurde auf $p<0,05$ festgelegt. Die Datenauswertung erfolgte mit der Statistik-Software IBM SPSS Statistics 24.

\section{Ergebnisse}

\section{Gewinnung und Merkmale der StudienteilnehmerInnen}

Insgesamt 106 der 1346 angefragten Arztpraxen (8\%) willigten zunächst in eine Studienteilnahme ein. Aufgrund von 35 nachträglichen Absagen der Beteiligung und Rekrutierungsproblemen in den teilnehmenden Praxen wurde die Rekrutierungszeit um 6 Monate verlängert. Von den in der Studie verbliebenen 71 Praxen (27 HausärztInnen und 44 OrthopädInnen) schlossen 36 Praxen schließlich 119 Reha-AntragstellerInnen ein. Nach Analyse fehlender Daten verblieben 83 Teilnehmende mit vollständigen Daten zu T1 und T0 in der Endstichprobe. 
Die Stichprobenbeschreibung ist ( $\triangleright$ Tab. 1). zu entnehmen. Der Antrag auf Rehabilitation wurde bei der Mehrheit der Stichprobe (84\%) bewilligt. Die Wartezeit auf den Rehabilitationsbescheid und somit der Zeitraum zwischen T0 und T1 betrug durchschnittlich $4,5( \pm 3,5)$ Wochen. Die Hauptgründe für die Beantragung der Rehabilitation waren aus Sicht der Betroffenen bei $77 \%$ der Stichprobe die körperlichen Beschwerden, bei $22 \%$ eine Kombination aus körperlichen und psychosozialen Belastungen. Mehr als ein Drittel (39\%) der StudienteilnehmerInnen hatte bereits in der Vergangenheit eine Rehabilitation aufgrund der MSK absolviert, $16 \%$ aufgrund einer anderen Erkrankung.

\section{Psychische Komorbidität bei Reha-Antragstellenden}

$69 \%$ der PatientInnen waren entsprechend der UKS-Auswertung [17] zu T0 in der Gruppe mit „moderater psychosozialer Belastung“, nur $23 \%$ „ganz oder eher unauffällig“ und $8 \%$ zu To als auch zu T1 jeweils „stark oder sehr stark belastet“ ( $\triangleright$ Abb. 1). Zu T1 verblieb die Mehrheit in der gleichen Belastungskategorie. Mehr als die Hälfte der Stichprobe (57\%) war weiterhin in der Gruppe mit „moderater psychosozialer Belastung“, $13 \%$ waren nicht mehr „moderat psychosozial belastet“, sondern in die Gruppe mit „ganz oder eher unauffälliger psychosozialer Belastung“ gewechselt $(p<0,001)$.

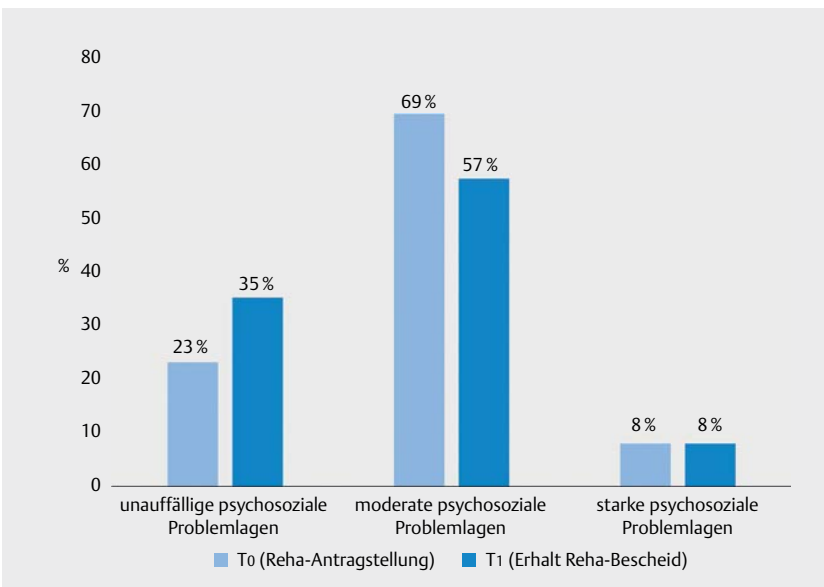

Abb. 1 UKS-Kategorisierung der Stichprobe zu To $(n=79)$ und T1 $(n=74)$.

\section{Psychometrische Prüfung des UKS bei Reha-Antragstellung}

Die Reliabilität (interne Konsistenz) der UKS-Gesamtskala weist Werte zwischen $\alpha=0,748$ (zu T0) und $\alpha=0,816$ (zu T1) auf. Für die UKS-Skala „Ängstlichkeit \& Depressivität“ (PHQ 4) lassen sich Werte zwischen $\alpha=0,696$ (zu T0) und $\alpha=0,813$ (zu T1) feststellen. Für die UKS-Skala „Schmerzen“ werden hohe Werte zwischen $\alpha=0,828$ (T0) und $\alpha=0,822$ (T1) erreicht. Die interne Konsistenz des UKS kann somit als mittel bis hoch beschrieben werden. Die Analyse der Mittelwerte des Gesamtscores des UKS zeigt, dass sich diese zu T0 und $\mathrm{T} 1$ nicht signifikant verändern $\left(\mathrm{M}_{\text {Diff }}=0,083 ; \mathrm{p}=0,770\right.$; $\mathrm{d}=0,039)$. Die Validitätsüberprüfung des Gesamtscores des UKS mit dem GSI (Global Severity Index) des BSI-18 ergibt zu T1 eine zufriedenstellende Übereinstimmung $\left(r_{\mathrm{tc}}=0,759\right)$.

\section{Zuweisungsdokumentation innerhalb der DRV}

Für 28 der 83 TeilnehmerInnen liegt eine interne Studiendokumentation von den PrüfärztInnen der DRV vor: 22 TeilnehmerInnen wurde die Rehabilitation bewilligt, am häufigsten die OR ( $n=17)$ gefolgt von der VMO ( $>$ Tab. 2). Eine Zuweisung in die psychosomatische Rehabilitation erfolgte nicht. Bei 6 Personen wurde die Leistung abgelehnt. Eine VMO wurde nur bei Antragstellenden befürwortet, von denen den PrüfärztInnen das UKS vorlag (IG) und darin psychosoziale Belastungen deutlich wurden: Dies betrifft 4 Personen mit „moderater psychosozialer Belastung“ und die einzige Person mit „starker Ausprägung“ psychosozialer Problemlagen. Von den 4 Antragstellenden der IG mit unauffälligen UKS-Werten wurden 3 der orthopädischen Rehabilitation zugewiesen und einmal der Antrag abgelehnt. Von den 17 Personen der IG mit „moderater psychosozialer Belastung“ erfolgte meistens eine Zuweisung zur orthopädischen Rehabilitation ( $n=11$ ), bei 4 Versicherten zur VMO und bei 2 Antragstellenden eine Ablehnung der Leistung.

\section{Arztbefragung zur Akzeptanz und Umsetzung des UKS}

Der Rücklauf der Arztbefragung zu T2 zur Akzeptanz und zu den Umsetzungsmöglichkeiten des UKS in der Praxis erreichte bei den 106 StudienärztInnen einen Anteil von 64\% $(n=68)$ und bei den 42 PrüfärztInnen der DRV einen Anteil von $66 \%(n=28)$.

Die StudienärztInnen wurden zunächst gebeten, die Anzahl ihrer Reha-Anträge bei erwachsenen MSK-PatientInnen im Jahr 2016 in

- Tab. 2 Zuweisungsdokumentation innerhalb der DRV [n (\%)].

\begin{tabular}{|c|c|c|c|}
\hline & Gesamt $(n=83)$ & KG $(n=36)$ & IG $(n=47)$ \\
\hline Zuweisungsinformation DRV liegt aus unbekannten Gründen nicht vor & $55(66 \%)$ & $30(83 \%)$ & $25(55 \%)$ \\
\hline Zuweisungsinformation DRV liegt vor & $28(34 \%)$ & $6(17 \%)$ & $22(45 \%)$ \\
\hline \multicolumn{4}{|l|}{ - davon Rehabilitation bewilligt: } \\
\hline Orthopädische Rehabilitation & $17(61 \%)$ & $3(50 \%)$ & $14(64 \%)$ \\
\hline Verhaltensmedizinisch-Orientierte Rehabilitation (VMO) & $5(18 \%)$ & - & $5(23 \%)$ \\
\hline Psychosomatische Rehabilitation & - & - & - \\
\hline - davon Rehabilitation abgelehnt: & $6(21 \%)$ & $3(50 \%)$ & $3(14 \%)$ \\
\hline
\end{tabular}


- Tab. 3 Reha-Antragstellung (im Jahr 2016) und Durchführung psychosozialer Screenings bei MSK-PatientInnen.

\begin{tabular}{|c|c|c|c|c|}
\hline & & HausärztInnen $(n=28)$ & OrthopädInnen ( $n=40)$ & $\mathbf{p}$ \\
\hline \multicolumn{2}{|c|}{$\begin{array}{l}\text { Anzahl der Anträge auf medizinische Rehabilitation in der } \\
\text { Zielgruppe für das Jahr } 2016[\mathrm{M}( \pm \text { SD)] }\end{array}$} & $18,8( \pm 13,4)$ & $38,8( \pm 28,9)$ & 0,001 \\
\hline \multirow{3}{*}{$\begin{array}{l}\text { Durchführung eines } \\
\text { standardisierten psycho- } \\
\text { sozialen Screenings für } \\
\text { MSK-PatientenInnen (n/\%) }\end{array}$} & ja & $1(4 \%)$ & $4(10 \%)$ & \multirow[t]{3}{*}{0,534} \\
\hline & bei Bedarf & $6(21 \%)$ & $10(25 \%)$ & \\
\hline & nein & 21 (75\%) & $26(65 \%)$ & \\
\hline
\end{tabular}

M = Mittelwert; SD = Standardabweichung; $n=$ Fallzahlen; \%= Prozent (Anteil an der jeweiligen Teil-/Gesamtstichprobe), $\mathrm{p}=$ Signifikanzniveau

Kostenträgerschaft der Rentenversicherung anzugeben und sich zur bisherigen routinemäßigen Integration psychosozialer Screenings zu äußern ( $>$ Tab. 3): HausärztInnen berichteten im Jahr 2016 für die MSK-Zielgruppe seltener Reha-Anträge [durchschnittlich $18,8( \pm 13,4)$ ] als OrthopädInnen [durchschnittlich 38,8 $( \pm 28,9)$ ] $(p=0,001)$. Fünf der 68 antwortenden StudienärztInnen (7\%) gaben an, dass sie ein standardisiertes psychosoziales Screening bei MSK-PatientInnen routinemäßig durchführen, ein Viertel (24\%) wendet dies bei Bedarf an. Die meisten Studienärztlnnen führen bei dieser Patientengruppe kein psychosoziales Screening durch.

Bezüglich der Akzeptanz des in der Studie eingesetzten Instruments für das psychosoziale Screening stimmte die Mehrheit aller StudienärztInnen völlig oder eher zu (am häufigsten die Hausärztlnnen mit 92\%), dass das UKS eine wichtige Zusatzinformationsquelle zum Befundbericht darstellt ( $\triangleright$ Abb. 2). Die Mehrheit der HausärztInnen (78\%), der OrthopädInnen (69\%) und PrüfärztInnen (61\%) stimmte außerdem der Aussage völlig oder eher zu, dass ein zukünftiger routinemäßiger Einsatz des UKS bei der Beantragung einer orthopädischen Rehabilitation zu befürworten sei (- Abb. 3). Des Weiteren wurden alle Arztgruppen hinsichtlich vier denkbarer Anwendungsszenarien des UKS im Rahmen der Reha-Antragstellung befragt ( Abb. 4): Lediglich $3 \%$ der OrthopädInnen und $4 \%$ der Prüfärztlnnen votierten dafür, das UKS den zuweisenden ÄrztInnen nicht vorzulegen und ausschließlich bei der Rentenversicherung auszuwerten (Szenario 1). Ein Großteil der StudienärztInnen und Prüfärztlnnen entschied sich dafür, dass sich die antragsinvolvierten ÄrztInnen das Screeningergebnis orientierend ansehen (Szenario 2) bzw. in Einzelfällen praxisintern auswerten können (Szenario 3), während die hauptverantwortliche Auswertung jeweils in der Rentenversicherung erfolgt. Mit $41 \%$ votierten HausärztInnen häufiger als OrthopädInnen (16\%) oder PrüfärztInnen (21\%) dafür, das Screening selbstständig im Rahmen der Befundberichterstellung auszuwerten und für die Rentenversicherung zu dokumentieren (Szenario 4).

\section{Diskussion}

Das zentrale Ziel dieser clusterrandomisierten, kontrollierten Studie war die Erprobung eines kurzen psychosozialen Screeningverfahrens (UKS) als ergänzendes Element der Reha-Antragstellung bei muskuloskelettalen Erkrankungen im Vorfeld der Rehabilitation.

Die Mehrheit der PatientInnen zeigte gemäß UKS-Auswertung eine „moderate“ (69\% zu T0 und 57\% zu T1) oder „starke psycho-

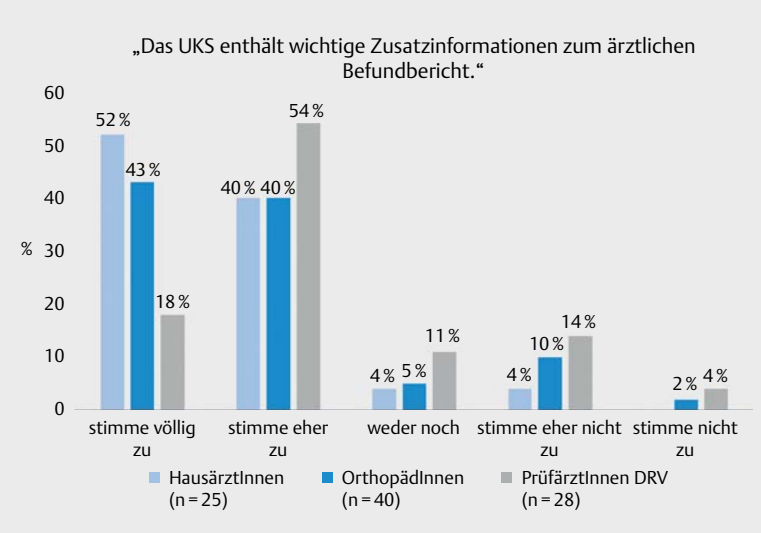

Abb. 2 Bewertung des UKS als zusätzliche Information im Befundbericht in Prozent (\%).

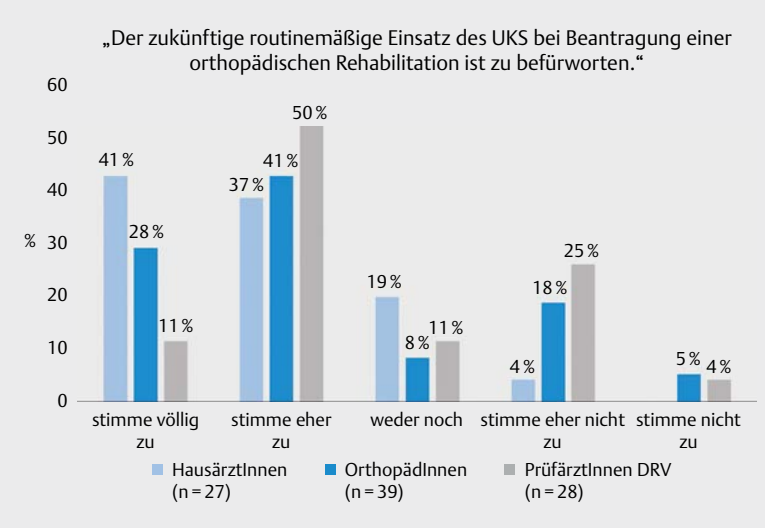

Abb. 3 Befürwortung eines zukünftigen Einsatzes des UKS in der Praxis in Prozent (\%).

soziale Belastung“ (T0 und T1: $8 \%$ ). Auch in einer Vorgängerstudie [7] konnte eine mit $60 \%$ ähnlich erhöhte psychische Belastung gemäß dem BSI-18 [21] zu Reha-Beginn bei RehabilitandInnen mit MSK der Region Mitteldeutschland festgestellt werden. Das UKS erfasst u. a. die Subskalen „Ängstlichkeit“, „Depressivität“ und „Schmerzbelastung“ der MSK. Im Vergleich mit dem BSI-18, der die Dimensionen „Ängstlichkeit“, „Depressivität“ und „Somatisie- 


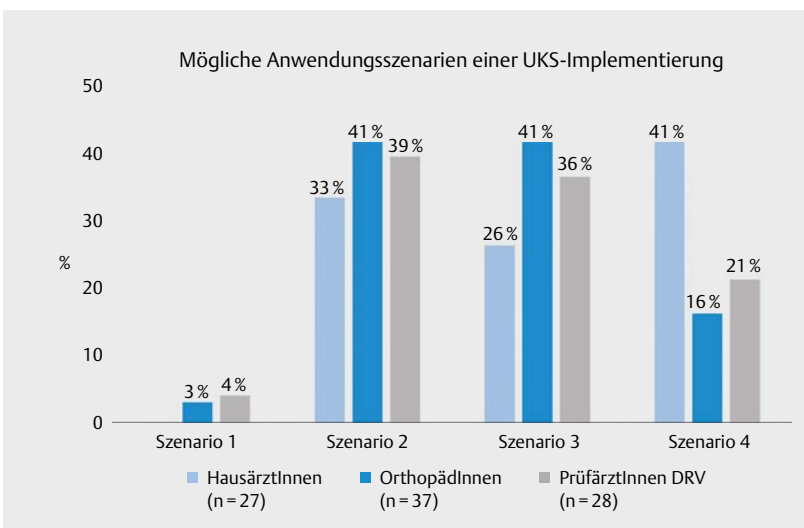

- Abb. 4 Zustimmung zu den 4 möglichen Anwendungsszenarien des UKS in Prozent (\%).

rung“ erfasst und eine Kategorisierung der Werte lediglich zwischen „auffälligen“ und „unauffälligen“ Personen zulässt [26, 27], differenziert das UKS, für das eine solche Aufteilung grundsätzlich im Rahmen einer alternativen Auswertungsvariante auch möglich ist, die Personen zusätzlich in eine mittlere Kategorie „moderate psychosoziale Belastung“ [20]. Die hohe Akzeptanzbeurteilung der Studien- und PrüfärztInnen gegenüber dem UKS lässt vermuten, dass die Befragten in diesem Screening eine potenziell wichtige Informationsquelle zu den üblichen oftmals rein medizinisch-somatischen Antragsunterlagen sehen. Dabei muss allerdings beachtet werden, dass das Feststellen einer behandlungsbedürftigen psychischen Störung weiterer, ausführlicherer Diagnostik bedarf. Für die auffallend hohe Anzahl an Personen der moderat belasteten Gruppe sind trotz Aufforderung zur konsekutiven Rekrutierung Selektionseffekte des Praxisteams im Sinne einer erhöhten Aufmerksamkeit und Ansprache hinsichtlich psychosozialer Problematiken der Teilnehmerlnnen nicht auszuschließen.

Die in dieser Studie festgestellte Anzahl von Personen mit „moderaten psychosozialen Problemlagen“ zum Zeitpunkt der Antragstellung (T0) erscheint außerdem mit 69\% deutlich höher als im bundesweiten Raum. Andere Studien zur psychischen Komorbidität in der somatischen Rehabilitation geben Prävalenzraten zwischen $31 \%[28,29]$ und $45 \%[30,31]$ an - bei muskuloskelettalen Erkrankungen vor allem für affektive Störungen (22,5-34,9\%) und Angststörungen (18,4-33,8\%) [6]. Zum Zeitpunkt des Erhalts des Reha-Bescheides (T1) kann ein Anteilsrückgang von $12 \%$ in der Gruppe mit moderaten psychosozialen Problemlagen festgestellt werden.

Nachgeschaltete Analysen zeigen, dass zwei Drittel dieser $12 \%$ unauffällige psychosoziale Problemlagen und ein Drittel starke psychosoziale Problemlagen zum zweiten MZP aufweisen. Da das UKS lediglich als Screeningverfahren angewendet werden kann, dem bei Auffälligkeiten eine ausführliche psychosoziale Diagnostik folgen sollte, lassen sich am ehesten individuelle Änderungen der Lebenssituation als Gründe für die Kategorienwechsel vermuten. Diese waren mit den im Projekt eingesetzten Instrumenten nicht hinreichend erfassbar.

Auch wenn in einer Vorgängerstudie für die Region Mitteldeutschland mit $60 \%$ eine ähnlich hohe Prävalenz psychischer Be- lastungen bei RehabilitandInnen mit muskuloskelettaler Indikation festgestellt werden konnte [10], ist in diesem Zusammenhang zu diskutieren, dass die Prävalenzen zahlreicher Studien in Abhängigkeit der jeweils eingesetzten Assessmentinstrumente variieren $[6,30]$. Auch Morfeld \& Friedrichs [32] weisen in ihrer Übersichtsarbeit darauf hin, dass die den Studien zu Grunde liegende Diagnostik wenig transparent und sehr heterogen sei. Gerade auch deshalb erscheint es sinnvoll, psychische Belastungen bei MSK-Indikation systematisch und assessmentbasiert im Rehabilitationskontext zu erfassen, um entsprechende weiterführende Diagnostik und Interventionen für Personen mit einem erhöhten psychosozialen Versorgungsbedarf zu initiieren [33].

Nachdem das UKS bisher v. a. im Rehabilitationssetting evaluiert wurde, konnte in dieser Studie bezüglich der psychometrischen Eigenschaften des UKS auch im Vorfeld einer Rehabilitation gezeigt werden, dass sich das Instrument grundsätzlich als ökonomisches und differenziertes Screeningverfahren zur Identifikation von psychosozialen Belastungen eignet. Die Werte zur Reliabilität und Validität der ersten 4 Items zur „Ängstlichkeit“ und „Depressivität“ des PHQ-4 wiesen bereits in einer repräsentativen Studie der deutschen Allgemeinbevölkerung ( $N=5.030)$ eine gute Screeningqualität auf [20]. Die Skala „Schmerzen“ erreichte ebenfalls gute Werte der internen Konsistenz. Der Analyse des Gesamtscores im Zeitverlauf von Reha-Antragstellung (T0) bis zum Erhalt des Rehabilitationsbescheides (T1) kann entnommen werden, dass sich die Mittelwerte in dieser Stichprobe nicht wesentlich verändern. Das spricht dafür, psychische Problemlagen im Vorfeld der Rehabilitation innerhalb dieses Zeitintervalls zu erheben. Die Items zur beruflichen und familiären Belastung ergänzen ebenso wie das Item zur Präferenz psychologischer Rehabilitationsinhalte, die üblichen Unterlagen zur Selbstauskunft und geben den niedergelassenen ÄrztInnen als auch den PrüfärztInnen der DRV eine assessmentbasierte Orientierung für die mögliche Bedeutung psychologischer Inhalte während der Rehabilitation.

Entsprechend sollte untersucht werden, wie häufig sich die PrüfärztInnen der DRV MD bei Bearbeitung der Reha-Anträge für die jeweiligen Rehabilitationskonzepte (OR vs. VMO vs. PR) entscheiden, wenn das UKS den Antragsunterlagen beiliegt und ob die vorliegenden Angaben des UKS für eine Entscheidungsfindung der Prüfärzte bei der Konzeptauswahl von Bedeutung sind. Aufgrund der zu geringen Fallzahlen musste auf die ursprünglich geplanten Berechnungen zur Überprüfung der getroffenen Zuweisungsentscheidung bzgl. des Rehabilitationskonzepts und deren Übereinstimmung mit dem UKS verzichtet werden. Konkrete Rückmeldungen aus Studienunterlagen lagen lediglich bei 28 Fällen vor. Ein Großteil (17 der 22 bewilligten Fälle) wurde einer orthopädischen Rehabilitation zugewiesen, von denen 11 Personen im UKS moderate psychosoziale Belastungen aufwiesen. Warum sich die PrüfärztInnen in diesen Fällen nicht für das VMO- Konzept entschieden, kann aus den vorliegenden Daten nicht abgeleitet werden. Nachgeschaltete Analysen zur Perspektive der Reha-Antragstellenden weisen eher in Richtung einer damit übereinstimmenden Zuweisung, da 10 dieser 11 Personen körperliche Beschwerden als Hauptgrund für die aktuelle Beantragung der Rehabilitation angaben. Inwieweit eventuell limitierte VMO-Kapazitäten in den Kliniken eine Rolle bei der Konzeptauswahl der PrüfärztInnen spielen, kann aus diesen Daten nicht beantwortet werden. VMO wurde bei fünf Per- 
sonen befürwortet, von denen das UKS bei Votierung der PrüfärztInnen vorlag und „moderate“ oder „starke psychosoziale Belastungen“ dokumentierte. Inwiefern das vorliegende UKS in der IG einen entscheidenden Einfluss bei der Zuweisung spielte, kann allerdings auch aufgrund der geringen Datenlage nicht beantwortet werden.

Auf eine gute Akzeptanz des psychosozialen Screenings mit dem UKS deutet die Zustimmung fast aller VertragsärztInnen und der Mehrheit der PrüfärztInnen, das UKS enthalte wichtige Zusatzinformationen zum ärztlichen Befundbericht, hin. Ebenso befürwortete ein Großteil der StudienärztInnen und die Mehrheit der PrüfärztInnen die zukünftige routinemäßige Implementierung bei der Reha-Beantragung. Nur $4 \%$ der HausärztInnen sahen das eher kritisch. Eine nähere Einordnung der von $29 \%$ der PrüfärztInnen geäußerten kritischen Einschätzung des zukünftigen UKS-Einsatzes ist auf Basis der geringen Rückläufe nicht möglich. Bei der Auswahl der vier Umsetzungsszenarien zeigte sich, dass die Mehrzahl der VertragsärztInnen die Möglichkeit haben möchte, das Screeningergebnis mindestens zu sehen bzw. in Einzelfällen selbstständig auszuwerten. Die hauptverantwortliche Auswertung soll aus Sicht der StudienärztInnen und der PrüfärztInnen bei der Rentenversicherung erfolgen. Bei praktischer Implementierung eines psychosozialen Screenings sollten die VertragsärztInnen die Möglichkeit zur selbständigen Auswertung haben, um zum einen für diese Aspekte im Kontext der Antragstellung auf Rehabilitationsleistungen sensibilisiert zu werden und zum anderen ggf. weitere notwendige diagnostische und therapeutische Schritte einzuleiten. Schließlich wird am häufigsten die finale Auswertung und Berücksichtigung des UKS durch die PrüfärztInnen der Rentenversicherung favorisiert.

\section{Limitierungen}

Die Aussagekraft und Generalisierbarkeit der Ergebnisse sind durch die Selektion der Praxen und PatientInnen stark eingeschränkt. Die gewünschte Fallzahl von 540 PatientInnen aus 130 Studienpraxen konnte trotz eines erheblichen Rekrutierungsaufwandes seitens des Studienzentrums nicht erreicht werden. Das bestätigen die auch in anderen Studien [34] aufgezeigten Grenzen der Projektdurchführung mit VertragsärztInnen trotz des geringen und gut strukturierten Dokumentationsaufwands sowie der Incentives für die Praxen. Von über 1340 kontaktierten Praxen konnten lediglich 71 ( 35 passive und 36 aktive) besonders engagierte Praxen für eine Studienteilnahme gewonnen werden. Die aus diesen 36 aktiven Praxen für die Studie eingeschlossenen PatientInnen mit 119 Anträgen in den 12 Studienmonaten entsprechen einer Aktivität von 0,28 Anträgen pro Praxis und Monat. Die Ergebnisse der Arztbefragung zu Reha-Antragszahlen bei MSK-Indikation für das Jahr 2016 zeigen allerdings, dass die kooperierenden Praxen durchschnittlich 2,56 Reha-Anträge/pro Monat in der studienrelevanten Patientengruppe mit MSK in Kostenträgerschaft der Deutschen Rentenversicherung stellten, davon maximal die Hälfte in regionaler Trägerschaft, entsprechend 1,3 Anträge pro Monat. Somit erreichte nur jeder fünfte der gestellten Reha-Anträge im Studienzeitraum das Forschungsinstitut. Es ist anzunehmen, dass der Aufwand für das Praxispersonal und/oder die PatientInnen sowie andere Gründe vielfach einer Studienteilnahme der Antragstellenden entgegenstanden. Möglicherweise sind in dieser Studie auch an anderer Stel- le gefundene grundsätzliche Vorbehalte von VertragsärztInnen gegenüber der medizinischen Rehabilitation, wie eine unzureichende Aussicht auf Erfolg des Reha-Antrages oder ein zu hoher Aufwand für die Erstellung des Befundberichtes, wirksam [35].

Vor allem aufgrund der geringen Praxen- und Patientenbeteiligung ist die ursprünglich geplante umfangreiche Evaluation mit randomisiertem kontrollierten Design zur Frage der UKS-Eignung für die optimierte Zuweisungssteuerung der Rehabilitationskonzepte gerade auch aus Sicht der PrüfärztInnen eingeschränkt. Außerdem konnte aufgrund des randomisierten Designs nicht jede(r) PrüfärztInnen der Rentenversicherung in den aktiven Prozess der studienbezogenen Bearbeitung eines Reha-Antrages mit vorliegendem UKS involviert werden.

Vor dem Hintergrund dieser Einschränkungen liefert diese Studie dennoch Hinweise auf die Integrierbarkeit des Kurz-Screenings auf psychosoziale Problemlagen im Rahmen der Reha-Antragstellung aus Perspektive der befragten Vertrags- und PrüfärztInnen und auf einen Zusatznutzen für die Zuweisungsentscheidung bezüglich bedarfsgerechter Angebote. Darauf zielt auch eine Integration psychosozialer Elemente in ein, allerdings aufwändigeres, Screening im Rahmen der Bedarfsfeststellung für Rehabilitationsleistungen der Deutschen Rentenversicherung Bund [36], deren Ergebnisse abzuwarten sind.

\section{FAZIT}

Psychosoziale Belastungen bei muskuloskelettalen Erkrankungen bilden eine zunehmende Herausforderung beim Zugang zu bedarfsgerechten medizinischen Rehabilitationsleistungen. Sie erfordern nicht erst innerhalb des rehabilitativen Versorgungssektors, sondern bereits bei Antragstellung und Zuweisung optimierte Prozesse. Gerade auch wegen des geringen Aufwands für die Routineversorgung in der primärärztlichen und orthopädischen Praxis wurde die Eignung des Ultra-Kurz-Screenings auf psychosoziale Belastungen für die Identifizierung entsprechender Problemlagen und Zuweisung zu Rehabilitationsangeboten untersucht. Es erscheint als sinnvoll, psychosoziale Problemlagen im Rahmen der Reha-Antragstellung standardisiert zu erfassen, damit behandelnden und antragsbearbeitenden ÄrztInnen diese zusätzliche Information bei Auswahl geeigneter Interventionen vorliegt. Auch wenn die geplanten Fallzahlen nicht erreicht werden konnten, sprechen die Ergebnisse für eine Akzeptanz des UKS durch die teilnehmenden Studien- und PrüfärztInnen und für die weiter wissenschaftlich zu begleitende Integration in die Antragsunterlagen sowohl zur besseren Orientierung der VertragsärztInnen als auch zur Unterstützung der Zuweisung seitens der Prüfärztlnnen.

Interessenkonflikt

Die Autoren geben an, dass kein Interessenkonflikt besteht. 
Literatur

[1] Raspe H. Gesundheitsberichterstattung des Bundes: Rückenschmerzen [Heft 53]. Berlin: 2012

[2] Schmidt CO, Raspe H, Pfingsten M et al. Back pain in the German adult population: prevalence, severity, and sociodemographic correlates in a multiregional survey. Spine 2007; 32: 2005-2001

[3] Raspe A, Matthis C, Héon-Klin V et al. Chronische Rückenschmerzen: Mehr als Schmerzen im Rücken. Ergebnisse eines regionalen Surveys unter Versicherten einer Landesversicherungsanstalt. Rehabilitation 2003; 42: 195-203

[4] Deutsche Rentenversicherung. Reha-Bericht 2018: Die medizinische und berufliche Rehabilitation der Rentenversicherung im Licht der Statistik. Berlin: 2018

[5] Härter M, Baumeister H, Reuter K et al. Increased 12-month prevalence rates of mental disorders in patients with chronic somatic diseases. Psychother Psychosom 2007; 76: 354-360

[6] Baumeister $\mathrm{H}$, Höfler M, Jacobi F et al. Psychische Störungen bei Patienten mit muskuloskelettalen und kardiovaskulären Erkrankungen im Vergleich zur Allgemeinbevölkerung. Zeitschrift für Klinische Psychologie und Psychotherapie 2004; 33: 33-41

[7] Beutel ME, Schulz H. Epidemiologie psychisch komorbider Störungen bei chronisch körperlichen Erkrankungen. Bundesgesundheitsblatt Gesundheitsforschung Gesundheitsschutz 2011; 54: 15-21

[8] Schmidt C. Der Einfluss psychischer Komorbiditäten auf den Bezug einer EM-Berentung nach medizinischer Rehabilitation. Rehabilitation 2016; 55: 284-289

[9] Schmidt C, Bernert S, Spyra K. Zur Relevanz psychischer Komorbiditäten bei chronischem Rückenschmerz: Häufigkeitsbild, Erwerbsminderungsrenten und Reha-Nachsorge im Zeitverlauf der Reha-Kohorten 2002-2009. Rehabilitation 2014; 53: 384-389

[10] Schmidt S, Parthier K, Mau W. Sozialmedizinischer und gesundheitsbezogener Verlauf von Rehabilitanden mit orthopädischen und psychosomatischen Erkrankungen unter Berücksichtigung bestehender Komorbiditäten. Akt Rheumatol 2017; 42: 151-161

[11] Deutsche Rentenversicherung. Verhaltensmedizinisch orientierte Rehabilitation: Anforderungsprofil der Deutschen Rentenversicherung Bund für die verhaltensmedizinisch orientierte Rehabilitation (VOR). Berlin: 2016

[12] Bethge M, Müller-Fahrnow W. Wirksamkeit einer intensivierten stationären Rehabilitation bei muskuloskelettalen Erkrankungen: systematischer Review und Meta-Analyse. Rehabilitation 2008; 47: 200-209

[13] Klesse C, Baumeister H, Bengel J et al. Somatische und psychische Komorbidität. Psychotherapeut 2008; 53: 49-62

[14] Härter M, Hahn D, Baumeister H et al. Komorbidität bei Patienten mit muskuloskelettalen und kardiovaskulären Erkrankungen. Journal of Public Health 2004; 12: 162-167

[15] Härter M, Woll S, Reuter K et al. Recognition of psychiatric disorders in musculoskeletal and cardiovascular rehabilitation patients 11 No commercial party having a direct financial interest in the results of the research supporting this article has or will confer a benefit upon the authors(s) or upon any organization with which the author(s) is/are associated. Archives of Physical Medicine and Rehabilitation 2004; 85: 1192-1197

[16] Reuter K, Woll S, Stadelmann S et al. Erkennen und Behandeln psychischer Belastungen und Störungen in der orthopädischen Rehabilitation. Zeitschrift für Klinische Psychologie und Psychotherapie 2002; 50: 313-327

[17] Baumeister $\mathrm{H}$. The importance of screening for depression in general practice. Acta psychiatrica Scandinavica 2011; 75: 75-76

[18] Jahed J, Bitz I, Bengel J et al. Dokumentation komorbider psychischer Störungen in der medizinischen Rehabilitation: Eine Analyse ärztlicher Entlassungsberichte. Rehabilitation 2012; 51: 385-391
[19] Schwarz S, Mangels M, Sohr G et al. Welche klinische Bedeutung hat die Vorabzuweisung von Patienten zur orthopädischen bzw. verhaltensmedizinisch-orthopädischen Rehabilitation durch den Rentenversicherungsträger? Rehabilitation 2007; 46: 323-332

[20] Küch D, Arndt S, Grabe A et al. UKS - Ultra-Kurz-Screening psychosozialer Problemlagen zur bedarfsorientierten Angebotszuweisung in der somatischen Rehabilitation. In: Arbeitskreis Klinische Psychologie in der Rehabilitation BDP., (Hrsg.). Psychologische Betreuung im KrankheitsverlaufDeutscher Psychologen Verlag; 2011

[21] Küch D, Becker J, Roßband J et al. Wie gut eignet sich der UKS als Screening-Instrument zur Erfassung psychosozialer Belastung? 22. Rehabilitationswissenschaftliches Kolloquium vom 4. Bis 6. März in Mainz. DRV Schriften 2013 Band 101

[22] Worringen U, Streibelt M, Schwabe M et al. Optimierung der Zuweisung zur verhaltensmedizinisch-orthopädischen Rehabilitation durch die Deutsche Rentenversicherung Bund: Erprobung eines Screening-Instruments. 21. Rehabilitationswissenschaftliches Kolloquium vom 5. bis 7. März in Hamburg. DRV Schriften 2012 Band 98

[23] Gräfe K, Zipfel S, Herzog W et al. Screening psychischer Störungen mit dem "Gesundheitsfragebogen für Patienten (PHQ-D)“. Diagnostica 2004; 50: 171-181

[24] Kroenke K, Spitzer RL, Williams JBW et al. An Ultra-Brief Screening Scale for Anxiety and Depression: The PHQ-4. Psychosomatics 2009; 50: 613-621

[25] Löwe B, Wahl I, Rose M et al. A 4-item measure of depression and anxiety: Validation and standardization of the Patient Health Questionnaire-4 (PHQ-4) in the general population. Journal of Affective Disorders 2010; 122: 86-95

[26] Franke GH, Jaeger S, Glaesmer $\mathrm{H}$ et al. Psychometric analysis of the brief symptom inventory 18 (BSI-18) in a representative German sample. BMC Med Res Methodol 2017; 14: doi:10.1186/s12874-016-0283-3

[27] Franke GH. BSI -Brief Symptom Inventory von L.R. Derogatis. Göttingen: Hogrefe; 2000

[28] Wunsch A, Rundel M, Härter M et al. Screening psychischer Störungen in der kardiologischen und orthopädischen Rehabilitation. Rehabilitation 2002; 41: 375-381

[29] Mohr B, Gräf T, Forster M et al. Der Einfluss von Depressivität und Geschlecht auf den Rehabilitationserfolg bei chronischem Rückenschmerz: Eine Pilotstudie. Rehabilitation 2008; 47: 284-298

[30] Baumeister H, Härter M. Psychische Komorbidität bei muskuloskelettalen Erkrankungen. Bundesgesundheitsblatt Gesundheitsforschung Gesundheitsschutz 2011; 54: 52-58

[31] Härter M, Baumeister H, Bengel J, Psychische Störungen bei Rehabilitanden mit einer somatischen Erkrankung.In: Härter M et al., (Hrsg.). Psychische Störungen bei körperlichen Erkrankungen. Heidelberg: Springer; 2007: 55-69

[32] Morfeld M, Friedrichs A. Psychische Komorbidität: Befunde zur Diagnostik und Hinweise auf Möglichkeiten der Weiterversorgung in der medizinischen Rehabilitation. Bundesgesundheitsblatt Gesundheitsforschung Gesundheitsschutz 2011; 54: 90-97

[33] Deutsche Rentenversicherung. Psychische Komorbidität: Leitfaden zur Implementierung eines psychodiagnostischen Stufenplans in der medizinischen Rehabilitation. Berlin: 2014

[34] Bleidorn J, Voigt I, Wrede J et al. Anrufen ohne Ende? Über das Gewinnen hausärztlicher Praxen für ein Versorgungsforschungsprojekt. Zeitschrift für Allgemeinmedizin 2012; 88: 61-68

[35] Schubert M, Fiala K, Grundke S et al. Der Zugang zu medizinischer Rehabilitation aus Perspektive niedergelassener Ärzte - Probleme und Optimierungsmöglichkeiten. Phys Med Rehab Kuror 2012; 22: 264-270

[36] Streibelt M, Kulisch K, Jaster M et al. Entwicklung eines Fragebogens zur Verbesserung der Informationen im Rahmen der Reha-Bedarfsfeststellung. 26. Rehabilitationswissenschaftliches Kolloquium vom 20. Bis 22. März in Frankfurt am Main. DRV Schriften Band 2017 Band 111 